The Egyptian Journal of Hospital Medicine (October 2019) Vol. 77 (3), Page 5276-5278

\title{
Evaluation of Serum Interleukin-6 Level in Vitiligo \\ Patients before and after Phototherapy
}

${ }^{1}$ Amr Mohamed Zaki, ${ }^{1}$ Hassan Mamdouh Abd El Aziz, ${ }^{2}$ Mamdouh Atiah Mohamed

${ }^{1}$ Ahmed Ayman Mohamed Abd El Hamed

${ }^{1}$ Department of Dermatology and Venereology, Faculty of Medicine - Al Azhar University

${ }^{2}$ Department of Clinical Pathology, Faculty of Medicine - Al Azhar University

Corresponding author: Ahmed Ayman Mohamed Abd El Hamed, Mobile: 01015896163

,email: ahmed.ayman4391190@gmail.com

\begin{abstract}
Background: Vitiligo is an acquired pigmentary disorder of the skin, resulting from a loss of functioning epidermal melanocytes. Multiple pathogenetic factors have been proposed, including the neural theory, genetic predisposition and impaired anti-oxidative defense. Objective: To evaluate the role of interleukin-6 in pathogenesis of vitiligo and the effect Narrow band-UVB on IL-6 serum levels. Patients and Methods: The study included 30 patients with vitiligo and 30 healthy controls. Each was submitted to thorough history taking, complete general, dermatological examinations including VASI score, and assessment of serum interleukin- 6 before and after 16 sessions of NB-UVB.

Results: Results of this study showed no statistical significant difference between patients and control as regard age and gender. Our study revealed a statistically significant difference between levels of IL-6 in patients group before and after therapy. Conclusion: Knowing exactly the role of IL-6 is important not only to clarify the pathogenesis of this disease, but also to improve the clinical course of vitiligo (i.e., disease stabilization and/or repigmentation) and the quality-of-life of patients.
\end{abstract}

Keywords: Broadband (narowband) UVB, systemic corticosteroids, erythrocyte sedimentation rate.

\section{INTRODUCTION}

Vitiligo is an inflammatory autoimmune skin disorder characterized by the progressive appearance of depigmented skin lesions caused by the loss of melanocytes at the cutaneous level ${ }^{(I)}$.

There is evidence suggestive that cytokines play a major role in autoimmune pathogenesis and promotion of disease activity. Many cytokines have been investigated in vitiligo, including IL-2, IL-4, IL17 , IL-6 TNF- $\alpha$, IFN- $\alpha^{(2,3,4,5,6)}$. Phototherapy has been the pillar of vitiligo treatment whether for stabilization or stimulation of repigmentation. Despite the emergence of different therapeutic tools, phototherapy remains significant. Limitations, related to absence of melanocyte reservoir, anatomic distribution, and duration of the lesions, might make this exceptional tool sometimes less effective. Phototherapy is still required with or resumed after surgical or medical correction in cases having such limitations ${ }^{(7)}$.

\section{AIM OF THE WORK}

The aim of the present study was to evaluate serum interleukin- 6 in vitiligo patients before and after treatment with phototherapy.

\section{PATIENTS AND METHODS}

This case control study included 30 patients presented with vitiligo (active-stable), diagnosed on the basis of typical clinical features, were selected as patient group. Thirty age and sex matched apparently healthy individuals were also included representing the control group. All patients were collected from the Outpatient Clinics of Dermatology and Venereology of Al-Azhar University Hospitals. An informed written consent was obtained from participant or their guardians before their participation in this study.

\section{Ethical Approval:}

Approval was obtained from Research Ethics Committee of faculty of Medicine, Al-Azhar University.

Patients

Inclusion criteria: Patients with any type of vitiligo. Older than 8 years. Patients had to stop topical therapy for vitiligo for at least 2 weeks before the study. And the systemic therapy at least 4 weeks before the study. Patients who can come regularly two times per week to have their phototherapy sessions.

Exclusion criteria: The patients had immunemediated comorbidities such as Graves' disease, insulin-dependent diabetes, atopic dermatitis or psoriasis or any other dermatological disease causing abnormal pigmentation. Pregnancy or lactation. Patients with phototoxic reactions related to phototherapy or photosensitivity or photomediated disorders as lupus erythematosus, albinism, or dermatomyositis, also patients with previous skin cancer or pre malignant skin lesions or taking immunosuppressive drugs such as methotrexate.

Controls: Healthy individuals with no symptoms or signs of vitiligo and with apparently healthy first degree relatives were selected as control group.

\section{Statistical analysis}

Data were analyzed using Statistical Program for Social Science (SPSS) version 15.0. Quantitative data were expressed as mean \pm standard deviation (SD). Qualitative data were expressed as frequency and percentage. 
RESULTS

Table (1): Demographic data of studied patients.

\begin{tabular}{|c|c|c|c|}
\hline \multicolumn{2}{|c|}{ Variables } & Patients $(\mathrm{N}=30)$ & Control $(\mathrm{N}=30)$ \\
\hline \multirow{2}{*}{ Age (years) } & Mean & 31.3 & 32.1 \\
\hline & \pm SD & 10.3 & 11.9 \\
\hline \multirow{2}{*}{ Gender } & Male & $11(36.7 \%)$ & $14(46.7 \%)$ \\
\hline & Female & $19(63.3 \%)$ & $16(53.3 \%)$ \\
\hline \multirow{3}{*}{ Clinical distribution } & Vulgaris & $16(53.4 \%)$ & --- \\
\hline & Segmental & $4(13.3 \%)$ & 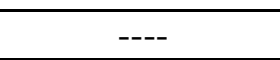 \\
\hline & Acro-facial & $10(33.3 \%)$ & $\begin{array}{ll}--- \\
-1\end{array}$ \\
\hline \multirow{2}{*}{ Family history } & Positive & $10(33.3 \%)$ & ---- \\
\hline & Negative & $20(66.7 \%)$ & ---- \\
\hline \multirow{4}{*}{$\begin{array}{l}\text { Duration of the disease } \\
\text { (years) }\end{array}$} & Mean & 10.37 & --- \\
\hline & \pm SD & 6.91 & ---- \\
\hline & Min & 0.5 & $\begin{array}{ll}--- \\
\end{array}$ \\
\hline & Max & 25 & --- \\
\hline \multirow{4}{*}{$\begin{array}{l}\text { Duration of last attack } \\
\text { (months) }\end{array}$} & Mean & 4.87 & $-\cdots$ \\
\hline & \pm SD & 3.70 & ---- \\
\hline & Min & 1 & --- \\
\hline & Max & 17 & --- \\
\hline \multirow{2}{*}{ Disease activity } & Stable & $18(60 \%)$ & $-\cdots$ \\
\hline & Active & $12(40 \%)$ & --- \\
\hline \multirow{2}{*}{ IL-6 (before) (pg/ml) } & Mean & 228.43 & 43.17 \\
\hline & \pm SD & 88.52 & 14.36 \\
\hline
\end{tabular}

Table (2): Comparison between patients and control as regard baseline IL-6.

\begin{tabular}{|c|l|c|c|c|}
\hline \multicolumn{2}{|c|}{ Variables } & Patients $(\mathbf{N}=\mathbf{3 0})$ & Control $(\mathbf{N}=\mathbf{3 0})$ & P-value \\
\hline \multirow{3}{*}{ IL-6 $(\mathbf{p g} / \mathbf{m l})$} & Mean & 228.43 & 43.17 & \multirow{3}{*}{$<0.001 *$} \\
\cline { 2 - 4 } & $\mathbf{\pm S D}$ & 88.52 & 14.36 & $(27-82)$ \\
\cline { 2 - 4 } & Range & $(102-430)$ & \\
\hline
\end{tabular}

This table shows highly statistical significant difference (p-value $<\mathbf{0 . 0 0 1}$ ) between patients and control as regard IL-6.

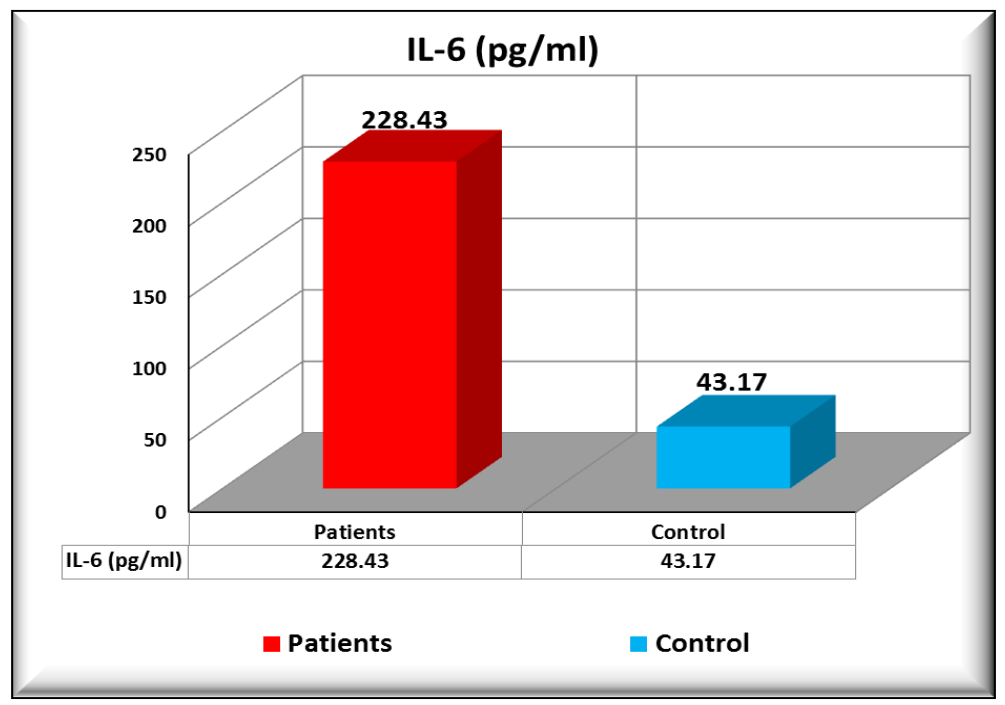

Figure (1): Comparison between patients and control as regard IL-6.

Table (3): Comparison between IL-6 in patients group before and after therapy.

\begin{tabular}{|c|c|c|c|c|}
\hline \multicolumn{2}{|c|}{ Patients group Variables } & Before $(\mathbf{N}=\mathbf{3 0})$ & After $(\mathbf{N}=\mathbf{3 0})$ & \multirow{2}{*}{ P-value } \\
\hline \multirow{3}{*}{ IL-6 (pg/ml) } & Mean & 228.43 & 112.10 & \multirow{2}{*}{ :0.001* } \\
\cline { 2 - 4 } & $\mathbf{\pm S D}$ & 88.52 & 45.69 & \\
\cline { 2 - 4 } & Range & $(102-430)$ & $(60-270)$ & \\
\hline
\end{tabular}

This table shows highly statistical significant difference (p-value $<\mathbf{0 . 0 0 1}$ ) between IL-6 in patients group before and after therapy. 


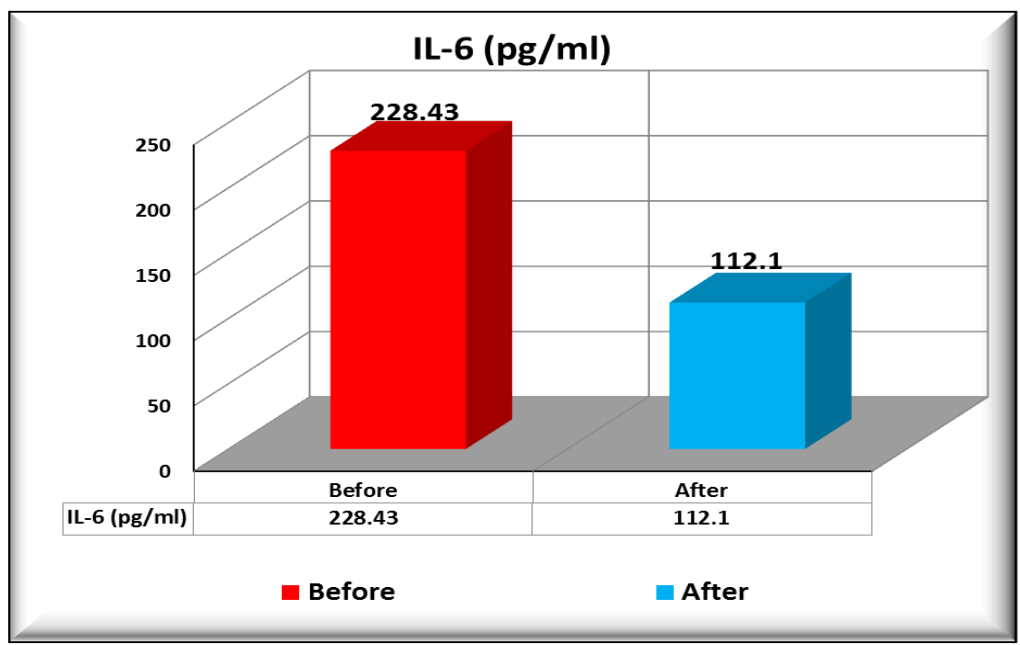

Figure (2): Comparison between IL-6 in patients group before and after therapy.

\section{DISCUSSION}

Vitiligo is an acquired disease with a variable course. It is characterized clinically by well-defined depigmented macules or patches thought to occur secondary to melanocyte dysfunction and loss ${ }^{(8)}$.

IL-6 act as a modulator of immune system by play a crucial role in the differentiation of dendritic cells (DCs). IL-6 produced by DCs, mainly plasmacytoid dendritic cells ( $\mathrm{pDCs}$ ), is critical for differentiation of B cells into plasma cells and production of antibodies ${ }^{(9)}$.

This case control study included 30 patients with vitiligo, and 30 age and sex matched healthy controls. NB-UVB treatment sessions were given for the patient group two times per week for 2 months. Serum level of IL- 6 was measured by ELISA before and after NB-UVB, to estimate its possible role in the pathogenesis of vitiligo and the effect of NB-UVB on IL-6. suggesting that local tissue secretes IL-6 into the circulation. These findings were consistent with $\boldsymbol{T u}$ et al. ${ }^{(10)}$; Pichler et al. ${ }^{(11)}$; who also found significantly higher serum levels of IL-6 in vitiligo patients as compared with healthy controls. However, there was no significant difference between active versus stable cases in our study.

$\underline{s)}$ and this comes in agreement with Albaaj et al. (12) who observed that there were no significant differences between male and females, active and stable cases or cases with different type of vitiligo regarding IL-6 and total antioxidant levels.

As we are the first study trying to demonstrate the effect of NB-UVB on serum. Finally, our study highlights the role of IL-6 in vitiligo pathogenesis and evaluation of its level before and after phototherapy.

\section{CONCLUSION}

The significant increase of serum levels of IL6 in vitiligo patients and the significant correlation between the level of IL- 6 and extension of vitiligo disease, clarify a positive feedback loop suggesting a positive systemic involvement of this cytokine in the pathogenesis of this disease. Moreover, the significant effect of NB-UVB treatment on serum levels of IL-6 in vitiligo patients, bring to the light that inhibition of IL6 could be a possible novel and promising therapeutic target of vitiligo disease and other autoimmune diseases.

\section{REFERENCES}

1. Ezzedine K and Silverberg $\mathbf{N}$ (2016): A practical approach to the diagnosis and treatment of vitiligo in children. Pediatrics, 138(1):e20154126.

2. Grimes PE, Morris R, Avaniss-Aghajani E et al. (2004): Topical tacrolimus therapy for Vitiligo: therapeutic response and skin messenger RNA expression of pro inflammatory cytokines.J Am Acad Dermatol., 51:52-61.

3. van den Boorn JG, Konijnenberg D, Dellemijin TA et al. (2009): Autoimmune destruction of skin melanocytes by perilesional T cells from vitiligo patients. J Invest Dermatol., 129:2220-32.

4. Bassiouny DA and Shaker O (2011): Role of interleukin-17 in the pathogenesis of Vitiligo. ClinExp Dermatol., 36:292-7.

5. Tembhre MK, Sharma A, Chattopadhyay S (2013): T helper and regulatory $\mathrm{T}$ cell cytokine profile in active, stable and narrow band ultraviolet $b$ treated generalized vitiligo. Clin Chim Acta., 424: 27-32.

6. Osman AM, Mukhtar MM, Bakheit KH et al. (2015): Plasma level of IL-17, IL-23 and transforming groth factor B in Sudanese Patients with Vitiligo: Acase control study. Indian J Dermatol., 60:635.

7. Esmat S, Mostafa W, Hegazy RA et al. (2016): Phototherapy: The vitiligo management pillar. Clinics in dermatology, 34(5):594-602.

8. Dillon AB, Sideris A, Hadi A et al. (2017): Advances in Vitiligo: An Update on Medical and Surgical Treatments. J Clin Aesthet Dermatol., 10(1):15-28.

9. Jego G, Palucka AK, Blanck JP et al. (2003): Plasmacytoid dendritic cells induce plasma cell differentiation through type I interferon and interleukin 6. Immunity, 19:225-234.

10. Tu CX, Gu JS, Lin XR (2003): Increased interleukin-6 and granulocyte-macrophage clony stimulating factor levels in the sera of patients with non-segmental vitiligoJournal of dermatological science, 31 (1): 73-78.

11. Pichler R, Sfetsos K, Badics B et al. (2009): Lymphocyte imbalance in vitiligo patients indicated by elevated CD4+/CD8+ T-cell ratio. Wien Med Wochenschr., 159: 337341.

12. Albaaj A, Foucras G, Raboisson D (2017): High somatic cell counts and changes in milk fat and protein contents around insemination are negatively associated with conception in dairy cows. Theriogenology, 88:18-27. 